\title{
Chart Parsing of Robust Grammars *
}

\author{
Sebastian Goeser \\ gsr@dhdibm1.bitnet \\ IBM Deutschland GmbH - GADL \\ Hans-Klemm-Str. 45 \\ D-7030 Böblingen
}

\section{Introduction}

Robustuess is a formal behnviour of naturnl language grammars to assign n best partial description to linguistic events whose strong description is inconsistent or cannot be constructed. Fvents of this sort may be called defective with respect to a grammar fragment. Defectiveness arises from the performance use that human beings make of langrage. Since defectiveness can be seen as failure of linguistic description, the principnl way to robustness is a method to wenken these descriptions.

Robust parsing, then, is parsing of robust granumars: a parser is robust iff it has the capability to interpret weak grammar fragments correctly. In this paper, I shall try to substnntiate this clnim by motivating n grammar dependent appronch to robust parsing and then describing $n$ chart parsing algorithm for ro. bust grammars. Though only c(ontext) $\mathrm{f}$ (ree) grammars will be adressed, there is an obvious extension of the nlgorithm to annotnted (unificntion-) granmars (WACSG formalism, see Goeser 1990) along the lines of (Shicber 1985).

Grammar based robustmess tools have been explored in a variety of formalisms, e.g. the metarule device within the ATN formalism (Weischedel and Sondheimer 1893), entity data structures in a case frame approach (Hryes 1984) or the weak description appronch in unification based grammars (Kudo et al. 1988, Goeser 1990). Parsing of grammars with ro-

\footnotetext{
${ }^{9}$ The work reported has been done while the author received an LGF gennt at the University of Stuttgntt.
}

bustness fentures competes with algorithmic approaches to robustness where parsing algorithrns, (usinnlly chnrt parsers except in Tomabechi and Tomitn (1988) where LR(k) parsing is advocated) are extended to include robustness features (Mellish 1989, Lang 1988) and/or heuristics to handle defect cases (Lnnger 1990, Stock et al. 1988).

Maybe the most critical issue in robust parsing is anbiguity, which emerges when constituency is loosened to some of substring analysis. E.g. Mellish (1989) parses for $n \operatorname{cfg} G$ the (cf) set $P A R(G)$ which is the set of all strings containing a sequence of nonempty substrings which is in the of language $L(G)^{j}$. In the worst case scenario where all these sequences are in $L(G)$, we get for a $w \in I(G)$ with an nmbiguity $k$ (in (r) en exponentinl nmbiguity of $k \times 2^{1 * 1}$ as an upper bound. Even in a non-worst case, which should be the case of renlistic cfgs, local ambiguities from substring analysis massively increase parsing time, E.g, in the (non-defective) example 1 , the arcs $a, b, c$ are empirically valid while the arcs $d, e$ nre artefacts of an algorithm parsing $P A R(G)$.

\footnotetext{
Sec Goeser (1990) for a more formsl discussion of $P A R(G)$.
} 
(1)

$\left.\begin{array}{lll}\text { Peter } & \bullet & \end{array}\right]$

Reflecting syutactic defectiveness in a cfg menus to nssign it a configurational regularity. Obviously, there is syntactic defectivity which is syntactically nonregulnr, such ns corrupted ontput from a speech recognition device (Tomnbechi and Tomitn 1988) ${ }^{2}$ or global constituent breaks (Goeser 1991), which can be subjected to syntactic prefix analysis only. On the other liand, there are spoken langunge constructions (Lindgren 1987, Goeser 1991, Janger 1990) and various kinds of "frngmentary utterances" (Carbonell and Hayes 1983) that definitively show configurational properties.

I,et us look nt a frequent spoken lnuguege coustruction called restart, as in the Gerinnn corpus exanple (2) ${ }^{3}$. Restarts follow a pattern $<\alpha \beta$ A $\beta^{\prime} \gamma>$ where the strings $\alpha$ and $\gamma$ but not $\beta$ and $\beta^{\prime}$ may be empty. The restart marker $\mathcal{A}$ is optional: in 67 from 96 restart samples $\beta$, which mostly cuds in a constituent break, and $\beta^{\prime}$ were separnted phonologically by tone constancy, a short pause or without any marking at all ${ }^{4}$. Restarts are $n$ kind of constituent coordinntion not allowing for ellipsis phenonent such as gapping, left deletion, split coordination or sluicing. The $\beta$ substring is usually defective und may indeed contnin arbitrary noise

\footnotetext{
T'This tmeterinl may show phonologicnl regulnrities, of couret

'All corpus ovidence reported here in puycliotherapeutic disconre from the ULMFIR TEX THANK

-Therefore, Langer's (1900) restart heuriatics secous empirically inndegunte innufar as it postulates a oyntnctic restnrt marker.
}

(see e.g. example (3)) ${ }^{5}$.

(2) da [ is es dnnm noch ein $\mathcal{A}$ there [is it then still a $\mathcal{A}$ $\alpha \beta$

kommt noch ein anderes Problem hinzu] comes yet nuotlier problem to-that] $\beta^{\prime}$

(3) der Peter [ hat konnte den dieses deshalb the Peter [ has could the this therefore

ehemaligen Lieferwagen

former truck.

A lat das gekenuft]

A has it bonght]

\section{Recursive partial string grammars}

Recursive partin] string grammins (RPSGs) are clgs with $n$ set of atnrt symbols and with rnles whose left hand side may be indexed with the keyword $S E T, S U B$, or PAR. The SET index on a rule' LHS licenses the adjunction of any start symbol to the right or left of its RHS string. The $S U B$ index licenses nrbitrary terminnl strings to the right or left of the indexed symbol's lexied projection. The PAR index includes $S U B$ and additionnlly licenses any terminal strings within this lexical projection. (Y,eft and right sided indices $S E T L, S U B L$ nnd $S E T R, S U B R$, respectively, are nlso in tuse). In n derivation relation $\Rightarrow$ for RPSGs nn indexed symbol $A_{4}$ unifies with category $A$ to give $A_{\eta}$. Formally, $S E T$ adjunction participates in the of dexivation relation, while $S U B$ and $P A R$ nre interpreted by a recursive generntion function gen operating on derivntions:

ger $_{\omega}: t \times\left(C_{\text {at }} \text { ind U Lex }\right)^{+}+\{(0,1)$

where $w$ is $n$ derivation, $t$ its tree structure, Catind the set of indexed or non-indexed nonternunals and $V_{e} x$ the set of terminals. The exnmple derintion tree (4) shows $S E T$ adjunction (dotted lines) and areas where arbitrary

"For a nore thorotygh diecusion of restart cyntax, ve Goener (1991). 
substrings are licensed by an indexed node. Generally, local arbitrariness within a string may be erily modelled with nn RPSG. Though firite cfls are turned into infinite ones through RPSG indexing, the syntactic description with RPSG is still configurational up to certnin local adjunctions.

\section{Basic algorithm}

As a parsing algorithu to start from, Earley's (1971) chart parser has been chosen, which has a top-down component adnptable to the top-down percolntion of index information, nud which guarnutees $n$ worst cnse complexity of $O\left(n^{3}\right)$ even for minximal ambiguity. We use the declarative Enrley variant in Dörre (1987). For n $\operatorname{cfg}_{\mathrm{B}} G=\left\langle C^{\prime}\right.$ at, Lex, $P$, S set $\rangle$, where Cat is $n$ set of non-terminals, Lez n set of terminals, $P$ a set of rules and $S$ set a set of start symbols, it is characturized by the following predictor concept:

- the predictor is $n$ relation $D(i, A) \subseteq$ $n^{+} \times C a l$ between $n$ vertex $i \leq n$ and a non-terminnl $\boldsymbol{A}$. It is integrated into the completer and scanner components (see below), 'This has the ndvantnge that no cyclic items i.e. items with an empty string of parsed symbols, hnve to be asserted to the chart.

- initialization is the specinl predictor case $D(0, S)$ where $S$ is a start symbol.

Let $V=$ Cat U Lex, $A \longrightarrow \alpha \beta \in P$ nnd $0 \leq i<j \leq n$. Chart $[i, j]$ be the set of arcs between vertices $i$ nud $j$ nnd $\stackrel{\star}{\Rightarrow}$ be the transjtive cover of the derivation relation. Then evcry item in the chart may be charncterized by the following membership condition ${ }^{6}$ which respects botll top-down (TD) and bottoin-up (BU) information. Remark that for the (basis variant of the) Earley algorithm, while item menbership depends on top-down predictor information, the acceptance of input strings is independent of the predictor (Kilbury 1985).

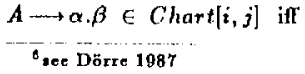

$[$ TD $] \exists S \in S$ set $S \stackrel{\star}{\Rightarrow} w^{\mathbf{0}, \dot{1}} A \delta \wedge$

[BU ] $\alpha \stackrel{\star}{\Rightarrow} w^{i, j}$

where $\delta \in V^{*}$

\section{The RPSG variant}

\subsection{Item Concept}

In the RPSG variant, items are represented as PROL,OG facts

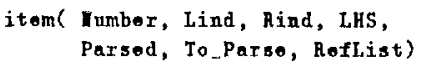

where iten number, the -possibly indexed- left hand symbol, the list of parsed symbols and the list of symbols yet to parse are well-known item parts. The variables Lind and Rind represent the status of substring generntion to the left and to the right of the Parsed string, respectively. Lind $\neq$ Rind is possible even for the $S U B$ index, since items represent prefix information on a constituent, wherens $n P A R$ index alwnys effects $L$ ind $=$ Rind. Partinl string information from higher nodes, which is justified only within the appropriate derivation, must be distinguisherl from $S U B$ or $P A R$ indexing of an iten's LHS symbol, which niways licences arbitrnry substrings. To allow reconstruction of a derivation, Reflist records the prirs of items (or pnirs of rule and item, see below) an item is coupleted from, or it equnls lex for lexicnl items ${ }^{7}$. To state the clinrt membership condition of the RPSG varinnt, we grveralize the function gen to an argument poir of strings of termituals and possibly indexed not-terminals:

$\operatorname{gen}^{\star}:\left(V_{i+1}^{+}\right)^{2} \rightarrow\{0,1\}$

where

$\operatorname{gen}^{*}(\alpha, \beta)=1$ iff $\beta$ can be generated from $\alpha$ $\left(\alpha, \beta \in V_{i, d}^{+}\right)$

The RPSG membership condition, then, is:

$$
\begin{aligned}
& A_{\eta} \rightarrow \alpha \cdot \beta \in \text { Chart }[i, j] \text { iff } \\
& { }^{7} \text { The Ref Liat in nlao used for paree forest construc- } \\
& \text { tion, see eg. Doerre (1987) for a diecus sion }
\end{aligned}
$$




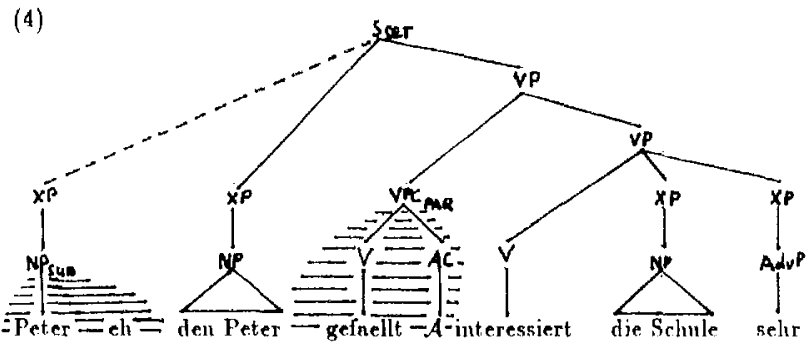

[TI] ] $\exists S \in S$ setind gen $\left(S, w^{n, i} A_{\eta} \delta\right)=1 \wedge$

[BU] $]$ ger1 ${ }^{*}\left(x, w^{i, j}\right):: 1$ or $\alpha=\epsilon$

where $\alpha, \beta, \delta \in\left(\dot{V}_{i n d}\right)^{*}$

\subsection{The Predictor}

The predictor of the RPSG varimit is, agail, a relation over vertices and non-ternumals. In contrast to the basis variant, lowever, a null predictor would be incorrect for the APSG vnrinnt, since the ncceptance of a string now depends on the substring information percolated by the predictor. The first predictor clanse allows an "initinlisation" for cuery vertex. The second clause formulates the expectation of n non-terminal $A_{\eta}$ by an acrive item i.e. nn item with n nonempty list To-Parse, and the third the expectation by pasive ilems with $n$ $S E T$ index. (Innse 4 expects a start symbol on the basis of left adjunction to $n, S E T$ indexed symbol. The following proposition, $n$ proof of which is avnilable from the nuthor, stntes the correctness of this predictor formalization.

$\operatorname{gen}^{\star}\left(S, w^{0, i} \Lambda, \delta\right)=1$ iff $D\left(i, A_{\eta}\right)$

for $n S \in S$ a $S t_{\text {indi }}$

\subsection{The Completer}

The completer component integrates the predictor relntion and the substring generation function nud lens two rules for rightside and

Aser Appendix $A$ for a complete formal eharncterination of the RPSG clint parser leftside ndjunction under a set-indexed symbol. Given that the conditions in the if-clnuse (and the looknliend condition, see below) yield, the conpleter adds new items to the chart ${ }^{9}$. Gluuse 1 of the RPSG completer, in, up to the generation function instend of derivntion, equivalent to the completer of the hasis variant: Given a rightside passive item, it adds a new item botl for a matching active item nnd for the prediction of an nppropriate rules's LHS symbol. Thus, no cyclic items have to be crented. Furthermore, since R.PSGs do not have productions, there is no need to handle cyclic items at nll. C'lause 2 does rightsidn ndjunction of a start symbol item to a passive $S E T$ indexed item. In left adjunction according to clnuse 3 , the adjoined (passive) item con agnin be licensed both by anotler (nctive or pasaive) $S E T$ indexed item or by the predictor relntion.

\subsection{Scamer and Lookahend}

Since the scnuner component ung be seen ns a lexical case of the completer, H. RPSG nigorithm could be reduced to n single active completer component and the controlling relntion $D$ (Kilbury 1985). Remark that the scanner allows for RPSG rules with RHS strings of terminals nud non-terminals. $\Lambda$ partinl looknhead of 1 , being npplied to nctive items only, has proven advnutageous in the besic variant (Dörre 1987). In the RPSG variaut, the length of the lookahend must be conditioned to the foct that zero or more non-derived but gencrated words miny follow a given vertex. The lookahend fails if, for the first To-Parse sym-

The relation $F$ includes the operation $\rightarrow$ which pro. cedurnlly nsserts new items to the chnrt 
bol, there is no first derivable lexical item, that is nccessible given the actual substring information.

Unfortunately, the scanner is not independent from this lookahead, since, in many cases, the item licensed by a lookahead operation onto n lexical item $i$ is exnetly the item licensing $i$ within the predictor relation. That is, from a procedural viewpoint of entering items into the chart, the lookahead condition and the predic. tor block ench other for certain lexical items. In this situation we decided to have a scanner withont a predictor relntion, thus paying for looknhend with an incrensed local lexical ambiguity.

\section{$5 \quad$ Status and Conclusion}

The algorithm described has been implemented and tested as part of the WACSG system that is based on the Stuttgart LFG system (Eisele 1987).

Chart parsing of robust ef grammars is n powerful method to cope with the configurational aspects of defectiveness. It is part of a major enterprise to re-analyze robustness not as a parsing problem but as a problem of weak lin. guistic description. Therefore, any formal work on the linguisties of defectiveness can be expected to improve our methods of robust parsing.

\section{Bibliography}

[1] Carbonell, J. and Hayes, P.: Recovery Strategies for Parsing Extragrammatical Language, in: AJCL 9, 3-4, 1983

[2] Dörre, J.: Weiterentwicklung des EarleyAlgorithmus für kontextfreie und ID/LP. Grammntiken, LiLog-Report 28, IBM Deutschland 1987

[3] Enrley, J.: An Efficient Context-free Parsing Algorithm, in: CACM 13, 2, 1970

[4] Goeser, S.: A linguistic Theory of Robustness, in: Proc. of COLING-13, Helsinki 1990
[5] Goeser, S.: Eine linguistische Theorie der Robustheit, Konstanz 1991

[6] Hnyes, P.J.: Entity-Oriented Parsing, in: COLING-10, Stanford 1984

[7] Kilbury, J.: Chart Parsing and the Earley nlgorithm, in: Klenk, U. (ed.): Kontextfreie Syntaxen und verwandte Systeme, Max Niemeyer, Tübingen 1985

[8] Kwasny, S.C. and and Sondheimer, N.K.: Relancention Techuiques for Pnrsing Grammatically Ill-Formed Input, in: AJCL 7,2, 1981

[9] Lnng, B.: Parsing Incomplete Sentences, in: Proc. COLING-12, Budapest 1988

[10] Lnnger, H.: Parsing Spoken Langunge, in: Proc. COLING-13, Helsinki 1990

[11] Mellish, C.S.: Some Chart-Based Techniques for parsing Ill-formed Input, in: Proc. ACL 27, Vanconver 1987

[12] Shieber, S.M.: Using Restriction to Extend Pnrsing Algorithms for Complex Fenture Bnsed Formalisms, in: Proc. ACI, 25, 1985

[13] Stock, O., Falcone, R., Insimnamo, P.: Island Parsing and Bidirectional Charts, in: Proc. COLING 12, Budapest 1988

[14] Tomabechi, H. and Tomita,M.: The Integration of Unification-Bnsed Pragmatics for Renl-Time Understanding of Noisy Continuous Speech Input, in: Proc. AAAI 7, Saint Pnul 1988.

[15] ULMER TEXTBANK: A mnchine-rendable corpus of spoken langunge from psychotherapentic discourse, University of Ulm

[16] Weischedel, H.M. nnd Sondheimer, N.K.: Metnrules as n Basis for Processing IIIFormed Input, in: AJCL 9, 3-4, 1983 


\section{Appendix}

Algorithm: An RPSG Chart Parser

Input:

1. RPSG $G=<$ Cat $_{\text {ind }}, L e x, P, S_{\text {set }}$ ind

2. string $w=w_{1}, \ldots w_{n}$

\section{Output:}

"nccepted", if $S \longrightarrow \alpha . \in C$ hart $|i, j|$ where

$S \in S_{\text {set }}$ ind and $\operatorname{gen}^{*}\left(\alpha, w^{0, n}\right)=1$

condition (predictor) :

Let $D\left(i, A_{\eta}\right) \subseteq n^{+} \times C \alpha t_{\text {ind }}$

$D\left(i, A_{\eta}\right)$ iff

1. $\exists S_{\zeta} \in S_{\text {set }}$ ind $_{\text {gen }}{ }^{*}\left(S_{\zeta}, g^{i} A_{\eta} \delta\right)=1$ or

2. $\exists C_{\zeta} \rightarrow \alpha . B_{\lambda} \beta \in C h a r t[j, k] \quad k \leq i \quad \wedge$ $\operatorname{gen}^{\star}\left(B_{\lambda}, g^{i-k} A_{\eta} \delta\right)=1$ or

3. $\exists C_{S K T} \longrightarrow \alpha . \in C h a r t[j, k] \quad k \leq i \quad \wedge$

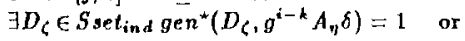

4. $\exists S_{\eta} \in S_{\text {set }}$ ind $\operatorname{gen}^{\star}\left(S_{\eta}, w^{0, i} C_{\zeta} \delta\right)=1 \wedge A_{\eta} \in S_{\text {setind }} \wedge \exists C_{S \boldsymbol{s}} \longrightarrow \beta \in P$

condition (lookahead) :

Let $F \subseteq \boldsymbol{P}^{\circ} \times n^{2}$.

$\mathrm{F}\left(C_{\eta} \rightarrow \alpha \cdot \beta^{\prime}, i, j\right)$ iff

1. $\left(\beta^{\prime}=\epsilon\right.$ or

$\beta^{\prime}=B \beta$ and $g e n^{\star}\left(B, g^{k-j} w^{k, k+1} \delta\right)=1$

for $\left.B \in C a t_{\text {ind }}, j \leq k \leq n\right)$ and

2. $C_{n} \longrightarrow \alpha \cdot \beta^{\prime} \mapsto$ Chart $[i, j]$ 
method:

- scanner: For $0 \leq i<j \leq n$ :

if $\quad B_{\zeta} \longrightarrow w^{i, i+1} w^{\prime} w^{j-1, j} \in P$ (where $w^{\prime} \in P P_{,+1}$ oder $w^{\prime}=\epsilon$ ) and $\operatorname{gen}^{\star}\left(B_{\zeta}, w^{i, j}\right)=1$,

then $\mathrm{F}\left(B_{\zeta} \longrightarrow w^{i, i+1} w^{\prime} w^{j-1, j}, i, j\right)$

- completer: For $0 \leq i<j \leq 1 \leq n$ :

1. if $\left(A_{\eta} \longrightarrow \alpha \cdot B \beta \in\right.$ Chart $[i, j]$ or

$D\left(j, A_{\eta}\right)$ and $A_{\eta} \longrightarrow B \beta \in \mathrm{P} \quad$ and $\left.\alpha=\epsilon\right)$ and

$B_{\zeta} \longrightarrow \gamma$. $\in$ Chart.jk,1] and $\operatorname{gen}^{*}\left(\alpha B_{6}, w^{i, 1}\right)=1$,

then $\mathrm{F}\left(A_{\eta} \rightarrow \alpha B_{\zeta}, \beta, i, 1\right)$

2. if $B_{\zeta} \longrightarrow \gamma$. Ghart $|k, 1|$ and $B \in$ Sset and

$A_{S B T} \longrightarrow \alpha, \in$ Chart $[i, j]$ and $\operatorname{gen}^{\star}\left(\alpha B_{\zeta}, w^{i, I}\right)$

then F $\left(A_{S E T} \rightarrow \alpha B_{\zeta, i, j}\right)$

3. if $A_{\eta} \longrightarrow \alpha$. $\in$ Chart $[\mathrm{i}, \mathrm{j}]$, and $A_{\eta} \in$ Sset and

$\left(B_{5 E T} \rightarrow \beta \cdot \gamma \in \operatorname{Chart}[k, 1]\right.$ or

$D\left(l, B_{S E T}\right)$ and $\beta=\epsilon$ and $\left.B_{S E T} \longrightarrow, \gamma \in P\right)$ and $\operatorname{gen}^{\star}\left(A_{\eta} \beta, w^{i, l}=1\right)$,

then $\mathrm{F}\left(B_{S B T} \longrightarrow A_{\eta} \mathcal{\rho} \cdot \gamma, i, 1\right)$ 